\title{
Effects of maternal age on embryo quality and pregnancy outcomes using testicular sperm with intracytoplasmic sperm injection
}

\author{
Hye Won Choi ${ }^{1}$, Yong-Seog Park', Sun-Hee Lee ${ }^{1,2}$, Chun Kyu Lim', Ju Tae Seo ${ }^{3}$, Kwang Moon Yang ${ }^{4}$ \\ 'Laboratory of Reproductive Medicine, Cheil General Hospital and Women's Healthcare Center, Dankook University College of Medicine, Seoul; ${ }^{2}$ Division \\ of Developmental Biology and Physiology, School of Biosciences and Chemistry, Sungshin Women's University, Seoul; ${ }^{3}$ Department of Urology, \\ ${ }^{4}$ Division of Reproductive Endocrinology and Infertility, Department of Obstetrics and Gynecology, Cheil General Hospital and Women's Healthcare \\ Center, Dankook University College of Medicine, Seoul, Korea
}

Objective: The aim of this study was to evaluate the influence of maternal age on fertilization, embryo quality, and clinical pregnancy in patients undergoing intracytoplasmic sperm injection (ICSI) using testicular sperm from partners with azoospermia.

Methods: A total of $416 \mathrm{ICSI}$ cycles using testicular spermatozoa from partners with obstructive azoospermia $(\mathrm{OA}, \mathrm{n}=301)$ and non-obstructive azoospermia (NOA, $n=115$ ) were analyzed. Female patients were divided into the following age groups: 27 to 31 years, 32 to 36 years, and 37 to 41 years. The rates of fertilization, high-quality embryos, clinical pregnancy, and delivery were compared across maternal age groups between the $\mathrm{OA}$ and NOA groups.

Results: The rates of fertilization and high-quality embryos were not significantly different among the maternal age groups. Similarly, the clinical pregnancy and delivery rates were not significantly different. The fertilization rate was significantly higher in the OA group than in the NOA group $(p<0.05)$. Age-group analysis revealed that the fertilization and high-quality embryo rates were significantly different between the $\mathrm{OA}$ and NOA groups in patients aged 27 to 31 years old, but not for the other age groups. Although the clinical pregnancy and delivery rates differed between the OA and NOA groups across all age groups, significant differences were not observed.

Conclusion: In couples using testicular sperm from male partners with azoospermia, pregnancy and delivery outcomes were not affected by maternal age. However, women older than 37 years using testicular sperm from partners with azoospermia should be advised of the increased incidence of pregnancy failure.

Keywords: Assisted reproductive technology; Intracytoplasmic sperm injection; Maternal age; Non-obstructive azoospermia; Obstructive azoospermia; Testicular sperm extraction

Received: Aug 25, 2016 · Revised: Oct 31, 2016 - Accepted: Nov 11, 2016 Co-corresponding authors: Kwang Moon Yang

Division of Reproductive Endocrinology and Infertility, Department of Obstetrics and Gynecology, Cheil General Hospital and Women's Healthcare Center, Dankook University College of Medicine, 17 Seoae-ro 1-gil, Jung-gu, Seoul 04619 , Korea

Tel: +82-2-2000-7788 Fax: +82-2-2000-7790 E-mail: km1yang@naver.com Yong-Seog Park

Laboratory of Reproductive Medicine, Cheil General Hospital and Women's Healthcare Center, Dankook University College of Medicine, 17 Seoae-ro 1-gil, Jung-gu, Seoul 04619, Korea

Tel: +82-2-2000-7590 Fax: +82-2-2265-5621 E-mail: arkangel@daum.net

This is an Open Access article distributed under the terms of the Creative Commons Attribution Non-Commercial License (http://creativecommons.org/licenses/by-nc/4.0/) which permits unrestricted non-commercial use, distribution, and reproduction in any medium, provided the original work is properly cited.

\section{Introduction}

With the advent of assisted reproductive technology (ART), intracytoplasmic sperm injection (ICSI) emerged as a breakthrough in the treatment of severe male infertility. Moreover, satisfactory fertilization and pregnancy rates have been achieved by testicular sperm extraction-intracytoplasmic sperm injection (TESE-ICSI) [1,2]. The outcome of TESE-ICSI can be influenced by various factors, including the etiology of azoospermia and the source of the retrieved sperm, in addition to female-related factors such as age and ovarian reserve [3]. The effect of age on fertility is of indisputable clinical relevance [4], and the partner's age is also regarded as an important criterion for 
predicting ICSI outcomes [5]. Similarly, maternal age is one of the most important factors influencing the likelihood of achieving a normal pregnancy by ART.

The effects of maternal age on pregnancy outcomes are well known, particularly the association of advanced maternal age with poor ART results [4,6-10]. Decreased ovarian reserve and decreased endometrial receptivity resulting from increased age are likely reasons for this observed reduction in fertility $[3,11]$, in addition to increased follicle disappearance beginning at the age of 37 years [1116]. In previous studies, significantly worse pregnancy results were achieved in subjects aged 40 to 49 years than in subjects aged $<40$ years $[17,18]$. Consistent with these results, Devroey et al. [13] reported delivery rates of $8.5 \%$ and $25.4 \%$ per embryo transfer (ET) in women older than and younger than 40 years old, respectively. Additionally, the chance of achieving a successful pregnancy has been shown to be low in women aged $\geq 41$ years [11]. However, maternal age has never been established as a main factor influencing pregnancy results in the most severe cases of male infertility. Similarly, the effects of oocyte and embryo quality (as determined by maternal age) on fertilization outcomes using testicular sperm have not been well established.

Therefore, the present study was performed to examine the possible relationship of clinical outcomes after ICSI with maternal age. This study also aimed to determine if oocyte and embryo quality, which are determined by maternal age, were associated with TESE-ICSI pregnancy results using sperm from partners with azoospermia.

\section{Methods}

\section{Patients}

From January 2008 through December 2013, to analyze the effects of age on pregnancy outcomes, 416 cycles of ICSI with the partner's own testicular spermatozoa were analyzed. Female patients were stratified into the following age categories: 27 to 31 years $(n=124$ cycles), 32 to 36 years ( $n=184$ cycles), and 37 to 41 years ( $n=108$ cycles). Since the number of cases was relatively low, patients less than 27 years and more than 41 years were excluded from this study. The rates of matured oocytes, fertilization, cultured high-quality embryos, transferred high-quality embryos, implantation, clinical pregnancy, and delivery were compared by maternal age between the obstructive azoospermia (OA, $n=301$ cycles) and non-obstructive azoospermia (NOA, $n=115$ cycles) groups. Male patients were evaluated, and azoospermia was classified as described previously [2,19]. Institutional review board approval was obtained for this study.

\section{Ovarian stimulation and oocyte retrieval}

Controlled ovarian hyperstimulation was performed in female pa- tients using a gonadotropin-releasing hormone agonist/antagonist protocol with human menopausal gonadotropin or recombinant follicle-stimulating hormone. Oocyte retrieval was performed via a transvaginal approach with sonographic guidance 36 hours after the administration of 10,000 IU of human chorionic gonadotropin (hCG) (Pregnyl, Organon, Haarlem, The Netherlands). The retrieved oocytes were incubated in G-Fert medium (Vitrolife, Kungsbacka, Sweden) supplemented with $10 \%$ human serum albumin solution (Vitrolife). Oocytes were maintained at $37^{\circ} \mathrm{C}$ in a $6 \% \mathrm{CO}_{2}$ atmosphere. At 3 to 5 hours after oocyte retrieval, cumulus cell masses were removed by incubating the cells for 1 minute in gamete medium supplemented with $0.05 \%$ hyaluronidase (Sigma, St. Louis, MO, USA).

\section{TESE and preparation}

The TESE and sperm preparation procedures have been described in detail elsewhere $[1,2,20]$. Briefly, testicular tissue was excised and rinsed with Quinn's Sperm Washing medium (SAGE, CooperSurgical, Trumbull, CT, USA). The tissue was squeezed with fine forceps, and the presence of spermatozoa was confirmed $(\times 200-\times 400$ magnification). The retrieved testicular spermatozoa were transferred to Gamete medium (Vitrolife) and maintained in an incubator at $37^{\circ} \mathrm{C}$ and $6 \% \mathrm{CO}_{2}$ until the ICSI procedure (approximately $3-5$ hours).

\section{ICSI procedure, assessment of fertilization, and embryo development}

Suspensions of testicular sperm were loaded into $10-\mu \mathrm{L}$ drops of gamete medium, and sperm motility was evaluated. Motile sperm were transferred to a droplet of $7 \%$ polyvinylpyrrolidone solution (SAGE) for immobilization, and individual oocytes were placed in droplets of buffered Gamete medium. After injection, the oocytes were washed and transferred to fertilization medium (G-FERT, Vitrolife). At 16 to 18 hours after ICSI, the oocytes were observed under an inverted microscope for any signs of damage due to the microinjection and for the presence of pronuclei. Normal fertilization was defined as the presence of two clearly visible pronuclei. Fertilized embryos were transferred to G-1.5 medium (Vitrolife). Embryo cleavage of the two-pronuclear oocytes was evaluated after 48 additional hours of in vitro culture (day 3). Embryos were assessed according to a previously described embryo grading system [1,2,21,22]. Embryos were graded according to the following criteria: grade I embryos, even blastomeres and no fragmentation; grade l-1 embryos, even blastomeres and $<25 \%$ fragmentation; grade II embryos, uneven blastomeres and <25\% fragmentation; grade II- 1 embryos, uneven blastomeres and $25 \%$ to $50 \%$ fragmentation; and grade III embryos, even or uneven blastomeres and $\geq 50 \%$ fragmentation. Cryopreserved pronuclear stage embryos were excluded from the total of cleavage-stage embryos. High-quality embryos were considered to 
be embryos of grade I, I-1, or II.

\section{ET and establishment of pregnancy}

The embryos were transferred into the uterine cavity 3 to 5 days after oocyte retrieval. Pregnancy was defined as a serum $\beta$-hCG level over $5 \mathrm{mlU} / \mathrm{mL}$ at 12 days after oocyte retrieval. Clinical pregnancy was defined as the presence of a gestational sac 5 to 7 gestational weeks after ET. The implantation rate was calculated as the number of gestational sacs observed divided by the number of transferred embryos. The pregnancy, clinical pregnancy, and delivery rates were calculated per complete ET cycle.

\section{Statistical analysis}

Statistical analysis was performed using SPSS ver. 12.0 (SPSS Inc., Chicago, IL, USA). Fertilization rates, numbers of cleavage-stage embryos, and numbers of high-quality embryos were compared among the three groups using one-way analysis of variance. Categorical data were analyzed using the Pearson chi-square test. Numerical data for the two groups of interest were compared by investigating their degree of correlation and performing the Student's $t$-test and chi-square test. Differences were considered statistically significant at $p<0.05$.

\section{Results}

A total of 6,099 stimulated oocytes were retrieved, and $76.5 \%$ of the matured oocytes were used to perform ICSI (Table 1). The male partner's age was significantly different among the age groups $(p<0.001)$. The fertilization and high-quality embryo rates were $71.5 \%$ and
$61.2 \%$, respectively. The clinical pregnancy rate was $37.1 \%$, and the delivery rate was $31.3 \%$. Among the maternal age groups, the fertilization and good embryo rates were not different. In the 27 to 31 years age group, the cultured high-quality embryo rate was lower than in the other groups, although this difference was not significant. The mean number of transferred embryos was $2.9 \pm 1.0$; this number was not significantly different among the age groups. The clinical pregnancy and delivery rates were lower in the 37 to 41 years age group than in the 32 to 36 years age group; however, this difference was not significant.

Both the male partner's age $(p<0.001)$ and the female partner's age $(p<0.05)$ were significantly different between the OA and NOA groups (Table 2). Moreover, the fertilization rate was significantly higher in the OA group than in the NOA group ( $75.2 \%$ vs. $61.8 \%, p<0.05)$. The total mean number of transferred embryos was $2.9 \pm 1.0$, which was also not different between the two groups ( $2.9 \pm 1.0$ vs. $2.9 \pm 1.0$ ). Furthermore, the clinical pregnancy and delivery rates were not significantly different between the OA and NOA groups (37.5\% vs. $35.8 \%$ and $31.3 \%$ vs. $31.6 \%$, respectively).

The influences of OA and NOA according to maternal age group are shown in Table 3. The male partner's age was significantly different between the 27 to 31 years and 37 to 41 years age groups $(p<0.05)$. The rates of fertilization, cultured high-quality embryos, and transferred high-quality embryos were significantly different in the 27 to 31 years age group $(p<0.05, p<0.001)$. In contrast, the other age groups showed similar results between the OA and NOA groups. However, in the 32 to 36 years age group, the implantation rates of the OA and NOA groups were significantly different $(p<0.05)$. Al-

Table 1. Effect of maternal age on outcomes in patients with azoospermic partners

\begin{tabular}{|c|c|c|c|c|}
\hline Variable & Total & $27-31 \mathrm{yr}$ & $32-36 \mathrm{yr}$ & $37-41 \mathrm{yr}$ \\
\hline Maternal age (yr) & $33.9 \pm 3.8$ & $28.9 \pm 1.3$ & $33.9 \pm 1.4$ & $39.1 \pm 1.4$ \\
\hline Paternal age (yr) & $37.3 \pm 5.9$ & $32.5 \pm 4.6^{\mathrm{a})}$ & $36.8 \pm 4.1^{\mathrm{a})}$ & $42.5 \pm 6.0^{a)}$ \\
\hline No. of cycles & 416 & 124 & 184 & 108 \\
\hline Basal FSH (mlU/mL) & $8.1 \pm 3.4$ & $7.1 \pm 2.5$ & $8.0 \pm 2.9$ & $9.3 \pm 4.5$ \\
\hline No. of retrieved oocytes & $14.7 \pm 10.5$ & $17.8 \pm 11.4$ & $15.0 \pm 9.9$ & $9.5 \pm 8.1$ \\
\hline Oocyte maturation rate (\%) & 76.5 & 76.5 & 75.9 & 78.2 \\
\hline Oocyte fertilization rate $(\%, n)$ & $71.5(3,238 / 4,591)$ & $70.3(1,232 / 1,753)$ & $71.3(1,455 / 2,040)$ & $74.7(596 / 798)$ \\
\hline High-quality embryo culture rate $(\%, n)$ & $61.2(1,427 / 2,330)$ & $57.3(470 / 820)$ & $61.7(635 / 1,030)$ & $67.1(322 / 480)$ \\
\hline No. of transferred embryos & $2.9 \pm 1.0$ & $3.0 \pm 0.9$ & $2.9 \pm 0.9$ & $2.9 \pm 1.1$ \\
\hline High-quality ET rate $(\%, n)$ & $83.4(887 / 1,063)$ & $83.8(249 / 297)$ & $82.3(391 / 475)$ & $84.9(247 / 291)$ \\
\hline No. of cycles with ET & 367 & 104 & 162 & 101 \\
\hline Pregnancy (\%) & 46.3 & 45.2 & 50 & 41.6 \\
\hline Clinical pregnancy (\%) & 37.1 & 35.6 & 40.7 & 32.7 \\
\hline Implantation (\%) & 17.7 & 18.5 & 18.9 & 14.8 \\
\hline Delivery (\%) & 31.3 & 32.7 & 34 & 25.7 \\
\hline
\end{tabular}

Values are presented as mean \pm standard deviation unless otherwise indicated. FSH, follicle-stimulating hormone; ET, embryo transfer. ${ }^{a)} p<0.001$. 
Table 2. Comparison of the influence of maternal age in the OA and NOA groups

\begin{tabular}{|c|c|c|}
\hline Variable & $\mathrm{OA}$ & NOA \\
\hline Maternal age (yr) & $34.2 \pm 3.9^{\mathrm{a})}$ & $33.1 \pm 3.7^{\mathrm{a})}$ \\
\hline Paternal age (yr) & $38.1 \pm 6.2^{\mathrm{b})}$ & $35.2 \pm 4.3^{b)}$ \\
\hline No. of cycles & 301 & 115 \\
\hline Basal FSH (mlU/mL) & $8.2 \pm 3.1$ & $7.8 \pm 4.2$ \\
\hline No. of retrieved oocytes & $14.4 \pm 11.1$ & $15.2 \pm 9.1$ \\
\hline Oocyte maturation rate (\%) & 76.6 & 76.4 \\
\hline Oocyte fertilization rate $(\%, n)$ & $75.2(2,501 / 3,325)^{a)}$ & $61.8(782 / 1,266)^{\mathrm{a})}$ \\
\hline High-quality embryo culture rate $(\%, n)$ & $61.2(1,063 / 1,737)$ & $61.4(364 / 593)$ \\
\hline No. of transferred embryos & $2.9 \pm 1.0$ & $2.9 \pm 1.0$ \\
\hline High-quality ET rate $(\%, n)$ & $83.6(658 / 787)$ & $83.0(229 / 276)$ \\
\hline No. of cycles with ET & 272 & 95 \\
\hline Pregnancy (\%) & 46.3 & 46.3 \\
\hline Clinical pregnancy (\%) & 37.5 & 35.8 \\
\hline Implantation (\%) & 18.5 & 15.9 \\
\hline Delivery (\%) & 31.3 & 31.6 \\
\hline
\end{tabular}

Values are presented as mean \pm standard deviation unless otherwise indicated.

$\mathrm{OA}$, obstructive azoospermia; NOA, non-obstructive azoospermia; $\mathrm{FSH}$, follicle-stimulating hormone; $\mathrm{ET}$, embryo transfer. ${ }^{\text {a) }} p<0.05 ;{ }^{\text {b) }} p<0.001$.

Table 3. Comparison of outcomes in the OA and NOA groups across different maternal age groups

\begin{tabular}{|c|c|c|c|c|c|c|}
\hline \multirow{2}{*}{ Variable } & \multicolumn{2}{|c|}{$27-31 \mathrm{yr}$} & \multicolumn{2}{|c|}{$32-36 \mathrm{yr}$} & \multicolumn{2}{|c|}{$37-41 \mathrm{yr}$} \\
\hline & $\mathrm{OA}$ & NOA & $\mathrm{OA}$ & NOA & $\mathrm{OA}$ & NOA \\
\hline Maternal age (yr) & $29.5 \pm 1.4$ & $29.4 \pm 1.1$ & $33.8 \pm 1.4$ & $34.1 \pm 1.5$ & $39.1 \pm 1.4$ & $39.1 \pm 1.7$ \\
\hline Paternal age (yr) & $34.3 \pm 5.1^{\mathrm{a})}$ & $31.7 \pm 2.9^{\mathrm{a})}$ & $37.0 \pm 4.6$ & $36.4 \pm 2.7$ & $43.0 \pm 6.1^{\mathrm{a})}$ & $39.8 \pm 4.8^{\mathrm{a})}$ \\
\hline No. of cycles & 81 & 43 & 131 & 53 & 89 & 19 \\
\hline Basal FSH (mlU/mL) & $7.4 \pm 2.8$ & $6.3 \pm 1.4$ & $7.8 \pm 2.8$ & $8.4 \pm 3.0$ & $9.3 \pm 3.4$ & $9.5 \pm 8.1$ \\
\hline No. of retrieved oocytes & $19.2 \pm 15.5$ & $17.9 \pm 9.20$ & $14.8 \pm 10.4$ & $15.4 \pm 8.7$ & $9.6 \pm 8.4$ & $8.7 \pm 6.6$ \\
\hline Oocyte maturation rate (\%) & 77.7 & 74.2 & 74.8 & 78.5 & 78.4 & 77.0 \\
\hline Oocyte fertilization rate $(\%, \mathrm{n})$ & $73.9(891 / 1,205)^{\mathrm{a})}$ & $\left.62.2(341 / 548)^{a}\right)$ & $75.9(1,100 / 1,449)$ & $60.1(355 / 591)$ & $76.0(510 / 671)$ & $67.7(86 / 127)$ \\
\hline High-quality embryo culture rate $(\%, n)$ & $58.8(339 / 577)^{\mathrm{a})}$ & $53.9(131 / 243)^{a)}$ & $61.2(462 / 755)$ & $62.9(173 / 275)$ & $64.7(262 / 405)$ & $80.0(60 / 75)$ \\
\hline No. of transferred embryos & $2.9 \pm 0.9$ & $2.8 \pm 1.0$ & $2.8 \pm 1.0$ & $3.1 \pm 0.8$ & $2.9 \pm 1.1$ & $2.6 \pm 1.2$ \\
\hline High-quality ET rate $(\%, n)$ & $87.7(185 / 211)^{b)}$ & $74.4(64 / 86)^{b)}$ & $81.5(269 / 330)$ & $84.1(122 / 145)$ & $82.9(204 / 246)$ & $95.6(43 / 45)$ \\
\hline No. of cycles with ET & 73 & 31 & 115 & 47 & 84 & 17 \\
\hline Pregnancy (\%) & 45.2 & 45.2 & 49.6 & 51.1 & 42.9 & 35.3 \\
\hline Clinical pregnancy (\%) & 34.2 & 38.7 & 42.6 & 36.2 & 33.3 & 29.4 \\
\hline Implantation (\%) & 17.5 & 20.9 & 21.5 & $13.1^{\mathrm{a})}$ & 14.6 & 15.5 \\
\hline Delivery (\%) & 31.5 & 35.5 & 33.9 & 34 & 48.9 & 17.6 \\
\hline
\end{tabular}

Values are presented as mean \pm standard deviation unless otherwise indicated.

$\mathrm{OA}$, obstructive azoospermia; NOA, non-obstructive azoospermia; $\mathrm{FSH}$, follicle-stimulating hormone; ET, embryo transfer.

${ }^{\text {a) }} p<0.05 ;{ }^{\text {b) }} p<0.001$.

though the clinical pregnancy and delivery rates were different between the OA and NOA groups in all of the age groups, significant differences were not observed.

\section{Discussion}

Prolonged life expectancy is associated with changing patterns of marriage and divorce. Specifically, remarriage and the wish to father a child in a new partnership are both becoming increasingly common [23]. Moreover, delaying childbirth has also become a trend and is especially common in developed countries, reflecting increased life expectancy and the changing role of women in society. However, as the ages of both partners increase, so do the risks of reproductive problems [24].

One of the most important factors influencing pregnancy success is maternal age [25]. Female fertility is well known to decrease as age 
increases [16,24]. Many reports have demonstrated the effects of aging on the ovarian response to stimulation and the fertilization rate. Aging has also been shown to contribute to decreased pregnancy and birth rates in assisted reproduction programs [26]. Advanced maternal age is associated with decreased numbers of retrieved oocytes [27] and reduced fertilization and implantation rates [28,29]. In addition, advanced maternal age has been shown to be associated with a decreased pregnancy rate, an increased spontaneous miscarriage rate, congenital anomalies, and a decreased delivery rate [24,30].

While female fertility peaks between the ages of 22 to 26 years, the age-related decrease in fertility first becomes prominent at the age of 35 years. This age is a discrete time point after which women exhibit significantly increased risks of adverse reproductive outcomes $[11,13,24,31-33]$. Previous studies have compared outcomes of women 40 years of age or older versus those of women younger than 40 years $[34,35]$. Another study demonstrated reduced fertility in women over 37 years of age [36]. By the age of 40 years, the likelihood of success for ART declines sharply [26,37], and the chance of a successful pregnancy is low in women aged $\geq 41$ years [11]. Therefore, we stratified patients by age into three groups (27-31 years, 32-36 years, and 37-41 years).

It has been postulated that the ovarian response to gonadotropin stimulation diminishes with increasing age. Decreased responsiveness of the ovary to gonadotropins, fewer collected eggs, and an increased cancellation rate are all associated with failure to achieve pregnancy [34,38]. Hughes et al. [39] suggested that early deterioration of granulosa cell function can be detected by a reduction in serum inhibin level; progressive follicular depletion and reduced granulosa cell function have also been suggested as factors contributing to the reduced fecundity observed with increasing age [26]. Female-related parameters, such as age and number of retrieved oocytes, may also have a significant contribution to the success of pregnancy [40]. From this perspective, Lee et al. [21] also reported that the female partner's age, number of matured oocytes, oocyte status, number of oocytes available for ICSI, and number of transferred embryos all affected in vitro fertilization outcomes. The observed decreases in ovarian reserve and endometrial receptivity are probable reasons for the reduced fertility associated with aging [11]. This decline becomes obvious at the age of 35 years. These effects can be explained partly by the age-related decrease in follicular reserve and the increased chromosomal anomaly rates in the oocytes [41], the latter of which is probably associated with the aging cytoplasm and increased aneuploidy rates during meiosis [42].

The deterioration in oocyte quality observed with increasing maternal age has been postulated as a contributing factor to reduced fertilization rates $[26,43,44]$. Moreover, poor oocyte quality has been shown to contribute to increased fetal loss in older women [35,44]. In our study, the matured oocyte and fertilization rates were not significantly different among the age groups. Additionally, maternal aging has been associated with increased spontaneous miscarriage rate, pregnancy complications, congenital anomalies, and higher perinatal mortality [24]. Delivery rates decrease by $50 \%$ for women aged 38 to 40 years and by another $50 \%$ by the age of 40 years [30]. In contrast, De Croo et al. [36] reported that maternal age less than 37 years did not influence fertilization or implantation results. Ishikawa et al. [3] reported that the pregnancy and delivery rates depended strictly on the age of the female partner, but not on her ovarian reserve or on the azoospermia prognosis. In addition, good fertilization rates were achieved without significant differences between the sperm sources. In our previous report [19], the overall fertilization rate per cycle was lower in the NOA group than in the OA group, but the embryo development and pregnancy rates per cycle did not differ between the $O A$ and NOA groups. However, in the present study, the cultured highquality embryo rate $(p<0.05)$ and transferred high-quality embryo rate $(p<0.001)$ were significantly different between the OA and NOA groups in women aged 27 to 31 years. This result suggests that even though the age of the couple was relatively young in this group, the diminished outcomes could have been affected by early and late paternal factors. These outcomes could also be attributed to poor embryo development due to deleterious factors specific to NOA.

Another important way in which the pregnancy rate can potentially be increased is by increasing the number of embryos transplanted into the uterus in older women $[32,45,46]$. The assisted reproduction outcomes of women aged 40 years and older could potentially be improved by transferring more than three oocytes to compensate for the poorer implantation rates [26]. However, we observed that the mean number of transferred embryos was $2.9 \pm 1.0$, and that this number was similar between the different age groups. Increasing the transfer of high-quality embryos with faster growth rates into the uterus is another strategy for potentially increasing pregnancy rates in older women [5]. Altay et al. [5] found no significant difference between the fertilization and cleavage rates of younger versus older women, but did detect a significant difference in the pregnancy rates of these two groups. We found that the clinical pregnancy and delivery rates declined from the 32-36 years to the 37-41 years group, but these differences were not significant. Since aging is a highly complex and incompletely understood process, clinicians often have difficulty engaging patients in a meaningful discussion of advancing maternal age [47]. Since the live birth rate is extremely poor in women 40 years of age and older, the possibilities of poor outcomes versus the best possible outcome should be explained to the couple before offering assisted reproduction to women of this age. Additionally, the possibility of using donor eggs should be discussed [26].

In conclusion, our data suggest that maternal age does not affect 
pregnancy or delivery outcomes for couples using testicular sperm from patients with azoospermia. However, women older than 37 years using testicular sperm from patients with azoospermia should be advised of the probability of poor pregnancy outcomes.

\section{Conflict of interest}

No potential conflict of interest relevant to this article was reported.

\section{Acknowledgments}

The authors wish thank to the staff of the Laboratory of Reproductive Medicine, Cheil General Hospital and Women's Healthcare Center, Seoul, Korea.

\section{References}

1. Park YS, Kim MK, Lim CK, Lee SH, Park DW, Seo JT, et al. Efficacy of cryopreservation of embryos generated by intracytoplasmic sperm injection with spermatozoa from frozen testicular tissue. J Assist Reprod Genet 2014;31:1331-6.

2. Park YS, Lee SH, Lim CK, Cho JW, Yang KM, Seo JT. Effect of testicular spermatozoa on embryo quality and pregnancy in patients with non-obstructive azoospermia. Syst Biol Reprod Med 2015; 61:300-6.

3. Ishikawa T, Shiotani M, Izumi Y, Hashimoto H, Kokeguchi S, Goto $\mathrm{S}$, et al. Fertilization and pregnancy using cryopreserved testicular sperm for intracytoplasmic sperm injection with zoospermia. Fertil Steril 2009;92:174-9.

4. Hassan MA, Killick SR. Effect of male age on fertility: evidence for the decline in male fertility with increasing age. Fertil Steril 2003;79 Suppl 3:1520-7.

5. Altay B, Kefi A, Tavmergen E, Cikili N, Semerci B, Tavmergen Goker $E$. The effects of female age on the outcome of testicular sperm extraction and intracytoplasmic sperm injection in infertile patients with azoospermia. Int Urol Nephrol 2002;33:95-9.

6. Battaglia DE, Goodwin P, Klein NA, Soules MR. Influence of maternal age on meiotic spindle assembly in oocytes from naturally cycling women. Hum Reprod 1996;11:2217-22.

7. Benadiva CA, Kligman I, Munne S. Aneuploidy 16 in human embryos increases significantly with maternal age. Fertil Steril 1996; 66:248-55.

8. Frattarelli JL, Miller KA, Miller BT, Elkind-Hirsch K, Scott RT Jr. Male age negatively impacts embryo development and reproductive outcome in donor oocyte assisted reproductive technology cycles. Fertil Steril 2008;90:97-103.

9. Whitcomb BW, Turzanski-Fortner R, Richter KS, Kipersztok S, Still- man RJ, Levy MJ, et al. Contribution of male age to outcomes in assisted reproductive technologies. Fertil Steril 2011;95:147-51.

10. Tsai YR, Lan KC, Kung FT, Lin PY, Chiang HJ, Lin YJ, et al. The effect of advanced paternal age on the outcomes of assisted reproductive techniques among patients with azoospermia using cryopreserved testicular spermatozoa. Taiwan J Obstet Gynecol 2013;52:351-5.

11. Ron-El R, Raziel A, Strassburger D, Schachter M, Kasterstein E, Friedler $\mathrm{S}$. Outcome of assisted reproductive technology in women over the age of 41. Fertil Steril 2000;74:471-5.

12. Gougeon A, Ecochard R, Thalabard JC. Age-related changes of the population of human ovarian follicles: increase in the disappearance rate of non-growing and early-growing follicles in aging women. Biol Reprod 1994;50:653-63.

13. Devroey P, Godoy H, Smitz J, Camus M, Tournaye H, Derde MP, et al. Female age predicts embryonic implantation after ICSI: a case-controlled study. Hum Reprod 1996;11:1324-7.

14. Faddy MJ, Gosden RG. A model conforming the decline in follicle numbers to the age of menopause in women. Hum Reprod 1996;11:1484-6.

15. Hull MG, Fleming CF, Hughes AO, McDermott A. The age-related decline in female fecundity: a quantitative controlled study of implanting capacity and survival of individual embryos after in vitro fertilization. Fertil Steril 1996;65:783-90.

16. Marcus SF, Brinsden PR. In-vitro fertilization and embryo transfer in women aged 40 years and over. Hum Reprod Update 1996;2: 459-68.

17. Flamigni C. Egg donation to women over 40 years of age. Hum Reprod 1993;8:1343-4.

18. Borini A, Bianchi L, Violini F, Maccolini A, Cattoli M, Flamigni C. Oocyte donation program: pregnancy and implantation rates in women of different ages sharing oocytes from single donor. Fertil Steril 1996;65:94-7.

19. Park YS, Choi SJ, Lee SH, Park DW, Lim CK, Jun JH, et al. Comparative results of embryo development and clinical pregnancy using sperm retrieved from fresh and frozen-thawed testicular tissue from patients with obstructive and non-obstructive azoospermia. Korean J Reprod Med 2009;36:301-10.

20. Park YS, Kim MK, Lee SH, Cho JW, Song IO, Seo JT. Efficacy of testicular sperm chromatin condensation assay using aniline blueeosin staining in the IVF-ET cycle. Clin Exp Reprod Med 2011;38: 142-7.

21. Lee SH, Song H, Park YS, Koong MK, Song IO, Jun JH. Poor sperm quality affects clinical outcomes of intracytoplasmic sperm injection in fresh and subsequent frozen-thawed cycles: potential paternal effects on pregnancy outcomes. Fertil Steril 2009;91: 798-804. 
22. Lee SH, Lee HS, Lim CK, Park YS, Yang KM, Park DW. Comparison of the clinical outcomes of day 4 and 5 embryo transfer cycles. Clin Exp Reprod Med 2013;40:122-5.

23. Kuhnert B, Nieschlag E. Reproductive functions of the ageing male. Hum Reprod Update 2004;10:327-39.

24. Dain L, Auslander R, Dirnfeld M. The effect of paternal age on assisted reproduction outcome. Fertil Steril 2011;95:1-8.

25. Malizia BA, Hacker MR, Penzias AS. Cumulative live-birth rates after in vitro fertilization. N Engl J Med 2009;360:236-43.

26. Dew JE, Don RA, Hughes GJ, Johnson TC, Steigrad SJ. The influence of advanced age on the outcome of assisted reproduction. J Assist Reprod Genet 1998;15:210-4.

27. Ziebe S, Loft A, Petersen JH, Andersen AG, Lindenberg S, Petersen $\mathrm{K}$, et al. Embryo quality and developmental potential is compromised by age. Acta Obstet Gynecol Scand 2001;80:169-74.

28. Szamatowicz M, Grochowski D. Fertility and infertility in aging women. Gynecol Endocrinol 1998;12:407-13.

29. Pantos K, Athanasiou V, Stefanidis K, Stavrou D, Vaxevanoglou T, Chronopoulou M. Influence of advanced age on the blastocyst development rate and pregnancy rate in assisted reproductive technology. Fertil Steril 1999;71:1144-6.

30. Ventura SJ, Mosher WD, Curtin SC, Abma JC, Henshaw S. Trends in pregnancies and pregnancy rates by outcome: estimates for the United States, 1976-96. Vital Health Stat 21 2000;(56):1-47.

31. Dor J, Seidman DS, Ben-Shlomo I, Levran D, Ben-Rafael Z, Mashiach $S$. Cumulative pregnancy rate following in-vitro fertilization: the significance of age and infertility aetiology. Hum Reprod 1996;11:425-8.

32. Widra EA, Gindoff PR, Smotrich DB, Stillman RJ. Achieving multiple-order embryo transfer identifies women over 40 years of age with improved in vitro fertilization outcome. Fertil Steril 1996;65:103-8.

33. Maheshwari A, Hamilton M, Bhattacharya S. Effect of female age on the diagnostic categories of infertility. Hum Reprod 2008;23: 538-42.

34. Wood C, Calderon I, Crombie A. Age and fertility: results of assisted reproductive technology in women over 40 years. J Assist Reprod Genet 1992;9:482-4.

35. Abdalla HI, Burton G, Kirkland A, Johnson MR, Leonard T, Brooks AA, et al. Age, pregnancy and miscarriage: uterine versus ovarian factors. Hum Reprod 1993;8:1512-7.
36. De Croo I, Van der Elst J, Everaert K, De Sutter P, Dhont M. Fertilization, pregnancy and embryo implantation rates after ICSI in cases of obstructive and non-obstructive azoospermia. Hum Reprod 2000;15:1383-8.

37. Schieve LA, Peterson HB, Meikle SF, Jeng G, Danel I, Burnett NM, et al. Live-birth rates and multiple-birth risk using in vitro fertilization. JAMA 1999;282:1832-8.

38. Bopp BL, Alper MM, Thompson IE, Mortola J. Success rates with gamete intrafallopian transfer and in vitro fertilization in women of advanced maternal age. Fertil Steril 1995;63:1278-83.

39. Hughes EG, Robertson DM, Handelsman DJ, Hayward S, Healy DL, de Kretser DM. Inhibin and estradiol responses to ovarian hyperstimulation: effects of age and predictive value for in vitro fertilization outcome. J Clin Endocrinol Metab 1990;70:358-64.

40. Silber SJ, Nagy Z, Devroey P, Camus M, Van Steirteghem AC. The effect of female age and ovarian reserve on pregnancy rate in male infertility: treatment of azoospermia with sperm retrieval and intracytoplasmic sperm injection. Hum Reprod 1997;12: 2693-700.

41. Munne S, Alikani M, Tomkin G, Grifo J, Cohen J. Embryo morphology, developmental rates, and maternal age are correlated with chromosome abnormalities. Fertil Steril 1995;64:382-91.

42. Martin RH. Meiotic errors in human oogenesis and spermatogenesis. Reprod Biomed Online 2008;16:523-31.

43. Segal S, Casper RF. The response to ovarian hyperstimulation and in-vitro fertilization in women older than 35 years. Hum Reprod 1990;5:255-7.

44. Meldrum DR. Female reproductive aging: ovarian and uterine factors. Fertil Steril 1993;59:1-5.

45. Grimbizis G, Vandervorst M, Camus M, Tournaye H, Van Steirteghem A, Devroey P. Intracytoplasmic sperm injection, results in women older than 39, according to age and the number of embryos replaced in selective or non-selective transfers. Hum Reprod 1998;13:884-9.

46. Lass A, Croucher C, Duffy S, Dawson K, Margara R, Winston RM. One thousand initiated cycles of in vitro fertilization in women> or=40 years of age. Fertil Steril 1998;70:1030-4.

47. Ramasamy R, Chiba K, Butler P, Lamb DJ. Male biological clock: a critical analysis of advanced paternal age. Fertil Steril 2015;103: 1402-6. 